\title{
Determinants of adherence in adults with cystic fibrosis
}

\author{
L J Kettler, S M Sawyer, H R Winefield, H W Greville
}

Thorax 2002;57:459-464

Cystic fibrosis (CF) is now as much a disease of adults as of children and adolescents. An important focus of recent research has been the impact of the complex, expensive, and time consuming routine of self-care and medical treatment that is required by adults with $C F$ to maintain health. A growing concern for medical and allied health teams is the issue of patient adherence to prescribed health management plans. A summary of the particular medical and treatment context of $\mathrm{CF}$ is followed by a review of adherence measurement issues and the determinants of adherence to treatment regimens in people with CF, primarily adults. Evidence for factors which influence adherence decisions of people with CF is examined. The medical and psychological aspects of this complex problem have not been adequately addressed because of difficulties with definition and measurement. Only a small proportion of the variance in adherence has been accounted for in the literature. New measurement technologies and new theoretical directions offer promise for a better understanding of this complex and important issue and may result in more effective intervention strategies to improve adherence.

See end of article for authors' affiliations

Correspondence to:

Correspondence to:
Ms L J Kettler, Department of Psychology, University of Adelaide, South Australia 5005, Australia; lisa.kettler@

psychology.adelaide.edu.au

Revised version received 12 October 2001 Accepted for publication 12 November 2001

\section{DEMOGRAPHIC DATA, AETIOLOGY, AND} TREATMENT OF CYSTIC FIBROSIS

Cystic fibrosis (CF) is the most common severe genetically inherited condition in the population of north west European descent, affecting approximately one in every 2500 live births. ${ }^{1}$ Significant advances in the management of respiratory infection and pancreatic insufficiency, coupled with better quality of care by specialist multidisciplinary teams, have resulted in a significant improvement in life expectancy to around 30 years. ${ }^{2}$ Preventative management and symptomatic treatment is instituted in early childhood for most people with CF, so management and treatment routines have been a daily concern for most adults with CF for many years.

People with CF have relatively good psychosocial health. ${ }^{3}$ The incidence of recognisable mental health disorders in people with CF is very similar to that in the general population. ${ }^{4}$ However, high levels of stress and, intermittently, high levels of distress are common and require recognition and attention. Moise et $a l^{5}$ noted higher self-esteem, lower levels of psychological distress, and better adaptation in patients who used avoidant coping strategies than in those who used more direct and positive coping methods. More recently, Abbott et al found that adults with the most serious disease more often reported their health to be better than others with CF. As will be discussed later, these findings raise questions about the interactions between treatment adherence and the use of coping strategies which depend on a high level of denial of the disease process and its threats to ongoing health and physical wellbeing.

Better life expectancy has brought with it the challenges of maintaining a complex and time consuming CF treatment regimen. ${ }^{7}$ For most people with $\mathrm{CF}$, management involves continuous home care with daily prophylactic medications such as oral or nebulised antibiotics, pancreatic enzymes, nebulised mucolytic agents, and vitamin supplements, as well as daily physiotherapy treatments. ${ }^{8}$ Ideal home care also involves optimising nutrition and exercise. From time to time most patients with CF require admission to hospital for intravenous antibiotics and intensive physiotherapy treatment of acute infective exacerbations of their lung disease. ${ }^{8}$ CF can also be complicated by diabetes ${ }^{9}$ and liver disease, conditions requiring substantial day to day management routines in their own right. There is also a growing concern about bone disease associated with $C F .{ }^{10}$ Lung transplantation does not reduce the requirement for ongoing self-care and close supervision.

In view of the complexity and extent of the treatment requirements for $\mathrm{CF}$, ongoing adherence to treatments is of interest and concern to CF clinicians.

\section{ADHERENCE TO MEDICAL REGIMENS}

Poor adherence to medical advice and treatment in chronic illness in general is well documented, with reports of patient adherence rarely exceeding $80 \%$ and more often falling between $30 \%$ and $70 \% .{ }^{11}$ The extent to which people adhere to recommended treatments appears to depend on the complexity and longevity of both the disease and its treatment. Research results are also influenced by the particular definition of adherent behaviour and the measurement strategies employed. ${ }^{12}$ In a substantial review of studies on life threatening disorders, ${ }^{13}$ the mean adherence rate for long term preventative regimens was found to be only $57 \%$ while the mean adherence rate for long term treatments was slightly less at $54 \%$.

The consequences of poor adherence-both for individuals and in terms of costs to the health system-are significant. ${ }^{14}$ Cluss and Epstein ${ }^{12}$ cited "exacerbation of disability, progression of the disease, more frequent medical emergencies, unnecessary prescriptions of more potent and/or 
toxic drugs and, ultimately, failure of treatment" to be important consequences of poor adherence to medical regimens and treatments.

Other reasons for examining patient adherence include quality assurance and accuracy in clinical trials ${ }^{11}$ and the collection of accurate data on the efficacy of ongoing treatments. The study of adherence may also allow us to gain a better understanding of patients' health beliefs and behaviour that hopefully will be a precursor to the design of better treatment programmes and improved relationships between patients and health professionals. This latter goal takes on particular significance in light of the dilemma which exists for health professionals between promoting good psychological health (and perhaps condoning and accepting denial and avoidance coping strategies) and promoting adherence, which is dependent on attention to and recognition of the disease process and the need to treat it.

\section{Definition of adherence}

The terms "compliance" and "adherence" are generally used interchangeably in the literature. However, Meichenbaum and Turk $^{11}$ argue that there is an important difference between them. They define compliance as "the extent to which patients are obedient and follow the instructions, proscriptions, and prescriptions of health care professionals", while adherence is defined as an "active, voluntary, collaborative involvement of the patient in a mutually acceptable course of behaviour to produce a desired preventative or therapeutic result". Although this is a useful definition and helps to conceptualise adherence, it does not assist in the establishment of criteria to define adherence behaviours-it does not tell us what someone must do to be considered adherent to a particular therapeutic or preventative regimen.

Many studies have found that substantially less than 100\% adherence is sufficient to result in desired health changes. Gordis $^{15}$ suggested that a suitable criterion for adherence behaviours is "the point below which the desired preventative or desired therapeutic result is unlikely to be achieved". Meichenbaum and Turk advocated the need to develop specific criteria based on particular conditions and treatments, rather than adopting general criteria for adherence behaviours. While this appears to be an acceptable criterion, it is only practical for those conditions where it is known how much of any prescribed behaviour is required to produce the desired effect. ${ }^{11}$ The chronic and variable nature of CF with its interdependent components of care and lack of knowledge of "how much is enough" for most aspects of the CF health care regimen make determination of such criteria an ongoing process of adjustment and balance.

Lask $^{16}$ suggested that patients should be described as fully adherent, partially adherent, or non-adherent-that is, he differentiated patient adherence using a quantitative approach. He noted that people may be adherent to some components of treatment and not others, and cautioned against the global labelling of patients as adherent or non-adherent. Koocher et $a l^{17}$ suggested that there are three different types of non-adherence: those who have inadequate knowledge, those who present psychosocial resistance, and those who are educatedly non-adherent - that is, have made an informed choice not to adhere. Lask ${ }^{16}$ classified non-adherent patients on the basis of their behaviour into three groups: "refusers" who say they don't want or don't need a particular treatment; "procrastinators" who are likely to say they will adhere more in future but never seem to get around to it; and "deniers" who will not admit to any non-adherence even when it is quite clear that their adherence is poor. Unfortunately, despite the recognition of various degrees and types of adherence, most of the literature reports adherence as a dichotomous constructeither adherent or non-adherent. We hypothesise that the majority of clinicians hold a similar view.
What emerges from these considerations is the importance of developing reasonable criteria for adherence which suit the specific context of patients' behaviour.

\section{Measurement of adherence}

The measurement of adherence is problematic. The most commonly reported techniques are to ask the patient, ask the physician, ask patients to keep a diary of their actions, count remaining pills, count the number of filled prescriptions, and review the medical record. All of these are indirect measures of the target behaviour and all are subject to problems of reporting bias, reporting errors, or intentional manipulation on the part of the reporter. Compared with more direct measurement techniques, all of the techniques described above have been shown to overestimate adherence. ${ }^{12} 18$

Another common approach involves drawing retrospective conclusions about adherence based on the therapeutic response, although this method carries substantial assumptions about the predictability and reliability of treatment responses to particular medications or other forms of treatment. ${ }^{18}$ When considering a complex disease such as CF, this method also forces assumptions about the interactions between different treatment elements and the stability of treatment effects for treatments other than the one targetedfor example, interactions between antibiotics and physiotherapy in the treatment of respiratory infections.

More direct measurement techniques have also been employed. In particular, blood serum levels or urinary excretion of medications, their metabolites, or of a tracer substance have been used to measure adherence with varying degrees of success. These methods have their own limitations, particularly the issue of pharmacokinetic variation. ${ }^{19}$ An additional concern with laboratory based measurements of adherence is the cost in time, money, and acceptability to the patient of collecting and analysing the data. Many blood and urine assays are only able to provide an accurate measure of the amount of medication consumed in the preceding 24 hours $^{20}$ so, while such a measure may provide information about the patient's adherence on the day before the test, daily testing would be needed to make an accurate assessment of adherence. Clearly, this kind of approach is invasive, expensive, and impractical for longer term treatment regimens.

There are a smaller number of reports of other more direct attempts to measure adherence behaviours using various electronic recording devices attached to pill bottles, aerosol dispensers (puffers), and nebulisers which record the date and time of each use of the dispenser. ${ }^{21-25}$ These data can be periodically downloaded for analysis and provide a more dynamic and longitudinal view of adherence than was previously possible. Researchers in this area report high levels of accuracy and reliability with these devices. ${ }^{22-25}$ The methodology offers advantages over both direct biological methods and diary reporting methods:

- The recording devices are non-invasive, relatively nonintrusive, and less dependent on patient cooperation for the collection of data.

- They allow for the continuous collection of data over a long period of time without the patient having to attend the clinic.

- They provide a measure of behaviour-not of belief, memory, or drug effect.

The main downside of these devices is their high unit cost price. They are, however, inexpensive to maintain and are usually reusable over a considerable period of time.

This type of monitoring has its own limitations. The data provide information about the use of a medication dispenser, not about whether the patient actually ingested the medication removed from the dispenser. However, it is reasonable to assume that most patients who make the effort to remove 
medication from the dispenser in the prescribed way (particularly over a long period of time) will also consume the medication. This technology is presently limited to medications and certain other specific activities (such as physiotherapy using a physiotherapy vest) and cannot provide us with information about adherence to treatment regimens such as exercise or diet. Researchers using these devices have also questioned whether this form of monitoring actually changes adherence behaviour-that is, whether the knowledge that their medication is being monitored causes patients to adhere better to the medication. This is an interesting question which holds both surprises and possibilities. It has been shown that, even when the monitoring has been explained to the patient, adherence behaviour is not significantly affected by monitoring alone $e^{21}$ and that, where monitoring does change adherence, the effect is very short lived..$^{183}$ Rand et al ${ }^{23}$ found that this form of monitoring allowed them to identify instances of medication "dumping" (the repeated use of the medication dispenser, far beyond prescribed levels, shortly before medical appointments) which could not be detected from other adherence measures, a finding that is inconsistent with the idea that monitoring itself improves long term adherence behaviour.

These findings may appear to be in contrast to the large body of psychological literature on the effectiveness of self-monitoring techniques in effecting behavioural change. ${ }^{26}$ Self-monitoring requires the patient to monitor and keep a written record of instances of a particular behaviour or emotion as part of a treatment programme, an approach used with many mental health disorders. It is known from this field of work that self-monitoring alone is usually insufficient to bring about desired changes in behaviour. The technique becomes effective with the addition of goal directed modifications of cognition and behaviour based on the self-monitoring observations. Of particular interest for the future are the opportunities presented by the merger of medication and health monitoring technologies and the methodology of selfmonitoring to assist behavioural change-for example, the use of blood glucose monitors by people with diabetes in assisting dietary management and diabetic control..$^{27}$

\section{PREDICTORS OF ADHERENCE IN ADULTS WITH CF}

People with CF often receive little positive reinforcement for their efforts to adhere to treatment. Furthermore, CF is an inexorable disease and, even with complete adherence, the health of an adult with CF will eventually decline. At best, good adherence is thought to reduce the rate of decline of respiratory disease. There is the real risk that adherence may be worse in those with the most severe disease because of the lack of positive reinforcement from any beneficial effect of treatment adherence. ${ }^{29}$

The treatment demands placed upon adults with CF are extraordinary when compared with most other chronic illnesses, let alone when compared with the healthy population. In addition to the complexity and number of treatments prescribed for adults with CF, adults living with this condition are faced with the challenge of interpreting and understanding the effects and priority of each of these treatments within their own treatment regime. It is not surprising that patients find this difficult, given the lack of consensus among treating physicians and multidisciplinary health care teams about which treatments are most important. ${ }^{730}$

People with CF often develop strong relationships with their multidisciplinary health teams. These relationships are usually developed over many years of treatment; CF is one of only a handful of chronic illnesses in which such long term treatment relationships may be developed. This feature of health care in CF will impact (both positively and negatively) on the communication between the health care professional and the patient. ${ }^{30}$ Such a relationship may enhance the clarity of the communication and may lend an important source of support to the patient. Importantly, though, it may also lead to assumptions on the part of both the health care professional and the patient about shared knowledge of the concerns to be dealt with and the treatment programme overall. The physician, physiotherapist, or dietician may assume, for example, that a patient knows how to manage a particular aspect of treatment if the subject has been discussed in a previous consultation. This assumption may be fair for much of the time, but there will be occasions when important information has not been exchanged which may result in adherence difficulties being unrecognised by both the CF team and the patient.

The disruption to longstanding health care relationships when older adolescents transfer their care from a paediatric to an adult facility must also be considered. ${ }^{31}$ The process of transfer can be an unsettling experience for some while, at the same time, it may herald a new sense of belonging or a fresh start. Important changes of this kind can impact on the dynamics of patients' self-perceptions and perceptions of place and belonging. ${ }^{32}$ In particular, transfer to an adult unit can signal a different level of parental involvement and supervision of the health care regimen for some young people. Changes in the dynamics of adherence may be predicted as part of this overall change process.

At present there is no information about the relationship between family functioning and either health outcomes or adherence in adults with CF. Unlike their healthy peers, more adults with CF remain within the family home or live alone than marry or share accommodation with others. ${ }^{3}$ It could be predicted that family functioning may be an important mediating variable for adherence for those adults who continue to live with immediate family and those who marry, but less so for adults with CF living independently. In this group, a broader definition of what constitutes family may be important to consider. The role of family cohesion, conflict, and stress have been linked to longer term trends in pulmonary functioning and weight gain for children and adolescents with $\mathrm{CF}^{33-35}$ While the mechanisms for these links remain unclear, ${ }^{7}$ it has been postulated that treatment adherence is the mediator of the effect, with those patients experiencing lower family stress, higher parental availability, and positive family coping adhering better to treatments and enjoying better health.

A limited number of studies have reported on adherence in adults with CF. Most are of an exploratory nature and employ self-reports, physician reports, or medical record reviews as their measurement strategy. As noted, these measurement strategies are problematic, raising concerns about the validity of the information which is likely to overestimate the extent of true adherence.

These early self-report and physician report studies indicate that the level of adherence to antibiotic treatments (oral or intravenous) is relatively high $(80-95 \%){ }^{36}$ This is a considerably higher rate of adherence to a longer term treatment than the figures previously found across a wide range of illnesses. ${ }^{13}$ Adherence to nebulised medications and pancreatic enzymes is reported to be moderate $(65-80 \%)$ which is also better than the figures reported more generally for long term regimens. However, adherence to vitamin therapy, dietary changes, exercise, and physiotherapy is generally reported to be poor (40$55 \%)^{37}$

These findings are consistent with the literature on adherence generally which suggests that adherence decreases as the duration and complexity of treatment increases. ${ }^{30}$ Interestingly, disease severity and general knowledge about CF are poorly associated with adherence, ${ }^{37} 38$ while worry about the condition and trust in medical practitioners appear to be related to greater levels of adherence. ${ }^{39}$ When asked specifically about why they do not adhere to treatments, adults with CF most often cite forgetfulness. ${ }^{36} 37$ 
There are significant limitations in the literature on adherence in adult patients with CF. In addition to the problems with measurement techniques, studies to date have been cross sectional in design and have presented a static picture of adherence. There has been no literature examining variability in adherence over time. Most studies report treatment factors (such as complexity and time) or trait factors (such as worry and confidence) as explanations for poor adherence. While these factors appear to have some explanatory value, most of the statistical effects are small, leaving significant amounts of the variance in adherence behaviour unaccounted for.

\section{MODELS OR THEORIES APPLIED TO ADHERENCE IN CF}

\section{Theories previously applied}

Efforts to understand adherence behaviour in CF through the application of theoretical models have met with limited success. The health belief model, ${ }^{40}$ the health locus of control construct, $^{41}$ and various models of coping ${ }^{42}$ have been applied. In addition, consideration has been given to the social cognitive theory of Bandura ${ }^{43}$ and, in particular, the concept of selfefficacy (the confidence to perform a particular behaviour).

Abbott $e{ }^{a} l^{39}$ found little support for the health belief model as a predictor of adherence in adults with CF. They were unable to discriminate between adherent and non-adherent patients on the basis of their health beliefs, with the exception of finding that those patients who worried more about their illness reported better adherence to most of their treatments. The results with the health locus of control model were more encouraging. Patients who believed that "chance factors" or "powerful others" controlled their health reported better adherence to physiotherapy regimens, enzymes and vitamin supplements, while those who believed they were in control of their own health reported better adherence to exercise regimens.

These findings warrant further investigation. Self-report of adherence behaviour was used in this study as the basis of testing the two theoretical models. However, it is likely that only a study using more objective measures of adherence will clarify the contribution of these theoretical models to the issue of adherence. It certainly seems reasonable to predict that patients who perceive their illness as serious, believe that treatment is beneficial, and are motivated or concerned for their health (as described in the health belief model) would adhere better to prescribed treatment programmes.

Czajkowski and Koocher ${ }^{42}$ explored the predictive value of six coping behaviours (understanding the severity of the illness, taking responsibility for medications at home, seeking information about the illness, future goal orientation, involvement in school or work, and openness with peers about illness) in distinguishing between adherent and non-adherent adolescents with CF. Reports of whether or not they used these coping behaviours reportedly discriminated between adherent and non-adherent patients; those who used the coping behaviours also had better adherence.

Parcel $e a^{44}$ evaluated the importance of various skills in maintaining adherence to the CF treatment programme. Skills included confidence in managing various aspects of medical treatment, symptom and behaviour monitoring, communication, and adjustment. They found that self-efficacy was the most important factor in predicting whether patients and their caregivers effectively monitored health and treated respiratory problems.

\section{New theoretical directions}

Previous studies ${ }^{13}$ suggest that the difficulties experienced by patients in achieving adherence are not disease specific, so it is appropriate to investigate models which have been applied to other diseases and in other disciplines in adults with CF. New models in health psychology offer unexplored possibilities for understanding the mechanisms which influence adherence to treatment in adults with CF. In particular, the self-regulatory model $^{45}$ has led to developments in the understanding and measurement of health behaviours including adherence to treatment. This model suggests that health related behaviours (including adherence) are influenced by the beliefs or cognitive schema (labelled "illness representations") which the patient holds about the illness. In this model it is considered that people structure their beliefs around the following five separate components or themes relating to the illness: "identity", "time line", "cause", "consequences", and "cure/ control".

A questionnaire measure was developed by Weinman $e t a l^{46}$ to measure patients' perceptions of their illness across these themes. Responses on this measure predicted patients' attendance for rehabilitation and their return to work following a myocardial infarction, ${ }^{47}$ suggesting a link between illness perceptions and adherence behaviours. The measurement of illness representations in adults with CF may assist us to predict the way in which they will adhere to their treatment and therefore to plan ways of managing the effects of illness representations on adherence.

Horne $^{48}$ examined beliefs about medicines using the concept of illness representations but targeting the treatment rather than the illness itself. It has been found that the beliefs that patients hold about the necessity of medications prescribed for them as well as specific concerns about potentially adverse effects of their medications account for a significant amount of the variance (15-20\%) in reported adherence to those medications. Across more than seven illness groups it was found that patients with a stronger belief about the necessity of their medication were more adherent than those with stronger concerns about potentially adverse effects. Patients who reported both a strong belief in the necessity of their medication as well as reporting a high level of concern about adverse effects were less adherent. It was hypothesised that such patients perform a "cost/ benefit analysis" of the pros and cons of taking medication, trying to minimise the perceived risks of the medication by taking less than the prescribed dose.

We propose that specific knowledge about a prescribed treatment may also influence the cost/benefit analyses performed by patients. It has been found that, although knowledge about CF in general has a very limited relationship with treatment adherence, there are strong relationships between accurate knowledge of a specific treatment regimen and better adherence to the regimen, at least among adolescents with $\mathrm{CF}^{49}{ }^{50}$

It seems therefore that the combination of better measurement techniques and appropriate theoretical models to guide our research questions may help us to predict (and test) more accurately the way in which people with CF adhere to their treatments.

\section{DIRECTIONS FOR FUTURE RESEARCH}

Adherence behaviour in patients with CF (particularly adults) is currently poorly understood and has been researched using exploratory and somewhat crude methodologies. We have not begun to understand the dynamics of symptom and treatment interactions which may guide the beliefs and decisions of adults with CF about how to adhere to their many and complex treatments, nor do we understand the processes and patterns which may be involved in treatment relationships between health professionals and patients over time. We know that many young people find it disruptive and unsettling to move from paediatric to adult services. We understand that people adhere with less consistency to more complex and time consuming treatments and we suspect that people who worry more about their disease adhere better to their treatments than those who are less worried. We have no information, 
however, about the way patients perform their treatments over time, and no accurate or objective information about how adherence to different treatment components relates to health outcomes in CF. People with CF are living longer, but we know that there is a high cost in both treatment demands and the development of additional symptoms in many cases.

If we are to develop our understanding of and ability to predict adherence to treatments in patients with CF (with the end goal of improving adherence), longitudinal studies of adherence behaviours are indicated with effective measurement tools. This is necessary as the current literature does not allow any understanding of the changing nature of adherence over time, with increasing age and maturity or disease severity. The complexity of the CF treatment regimen also provides an important opportunity to study adherence behaviour concurrently within multiple different management regimens (e.g. physiotherapy, antibiotics and vitamins). These studies are necessary if we are to gain an understanding of the relationship between adherence and outcome in CF.

There are additional research challenges in relation to adherence which face the CF community. The issue of adequate criteria for adherence, both from a clinical and a research point of view, remains unresolved. Internationaland, in many cases, national-agreement about minimal standards of care in CF is yet to be achieved, together with an understanding within different treatment regimens of how much is enough. These issues make discussion about adherence criteria fraught with uncertainty. We have a poor understanding of the different adherence challenges faced by patients with CF who have different levels of disease severity and who are at different life stages. What may be true for one patient at a particular stage of life may not be true or appropriate for another at a different developmental stage. It is likely that qualitative studies will provide a deeper understanding of these issues.

On a broader level, CF and other chronic illnesses pose a challenge for those training doctors and other health care professionals. Training in most disciplines relevant to CF is focused on the treatment of acute illnesses (or a series of acute illnesses), a very different proposition from the long term management of people with a chronic illness. ${ }^{51}$ In addition, physicians receive limited training in communication skills and in strategies that may enhance the quality of communication within a consultation. The lack of knowledge about how adherence can be improved means that most health professionals receive little specific training about the simple communication strategies that can promote better adherence to the health care regimen. ${ }^{52}$

We have begun to investigate the links between patient beliefs and perceptions of their disease and its treatment and their adherence to those treatments. We have conducted a cross sectional pilot study to assist with the validation of a new questionnaire measure of beliefs and perceptions about $\mathrm{CF}$ and its treatment and are now using electronic monitoring technology to collect detailed information from adults with CF using two different medications over a period of 3 months. ${ }^{53}$ This will allow us to examine the relationship between patterns of adherence to those medications and patient beliefs about their treatments. It is hoped that a better understanding of treatment adherence in adults with CF will lead to improvements in treatments, health outcomes, and patient quality of life.

Few adherence interventions have been tested in patients with CF-whether children, adolescent, or adult. We believe that only with greater understanding of the complex issue of adherence to treatment in people with CF (and other chronic conditions) will we be able to design and test interventions that aim to improve and maintain adherence in order to improve health and wellbeing.

\section{Key points}

- There is an increasing recognition among health care professionals that managing and promoting adherence to treatment is an essential component of CF care.

- Previous research indicates that patients with CF adhere more to some treatment components than others, and adhere to some treatments less than $50 \%$ of the time.

- Exploration of the issue of adherence in CF has been hampered by unclear criteria for adherence and limitations in measurement techniques.

- Electronic monitoring devices, in combination with longitudinal research designs, offer the promise of more valid and reliable information about rates and patterns of adherence in CF.

- Theoretical models such as the self-regulatory model, drawn from health psychology, may assist us to develop a more sophisticated understanding of adherence behaviours among patients with $\mathrm{CF}$.

- Interventions for the promotion of adherence in CF must be developed with sensitivity for the individuals living with this complex and progressive disease, with its extraordinary treatment demands and lack of clarity about clinical best practice.

\section{Authors' affiliations}

L J Kettler, H R Winefield, Department of Psychology, University of Adelaide, Adelaide, South Australia 5001

S M Sawyer, Centre for Adolescent Health and Department of

Respiratory Medicine, Royal Children's Hospital, Parkville, Victoria 3052, and Department of Paediatrics, University of Melbourne, Victoria,

Australia

H W Greville, Adnet CF Unit, Department of Thoracic Medicine, Royal Adelaide Hospital, Adelaide, South Australia 5000

\section{REFERENCES}

1 Sharp C, McNeil R, Wales S, et al. Young adults with cystic fibrosis: social well-being and attitudes. Aust Nurses J 1994;2:38-40.

2 Elborn S. The management of young adults with cystic fibrosis: 'genes, jeans and genies'. Disabil Rehabil 1998;20:217-25.

3 Shepherd SL, Hovell ML, Harwood IR, et al. A comparative study of the psychosocial assets of adults with cystic fibrosis and their healthy peers. Chest 1990;97:1310-6.

4 Blair C, Cull A, Freeman CP. Psychosocial functioning of young adults with cystic fibrosis and their families. Thorax 1994;49:798-802.

5 Moise J, Drotar D, Doershuk C, et al. Correlates of psychosocial adjustment among young adults with cystic fibrosis. J Dev Behav Pediatr 1987;8:141-8.

6 Abbott J, Dodd M, Webb AK. Different perceptions of disease severity and self care between patients with cystic fibrosis, their close companions, and physician. Thorax 1995;50:794-6.

7 Quittner AL, Drotar D, levers-Landis C, et al. Adherence to medical treatments in adolescents with cystic fibrosis: the development and evaluation of family-based interventions. In: Drotar D, ed. Promoting adherence to medical treatment in chronic childhood illness: concepts, methods and interventions. Mahwah, NJ and London: Lawrence Erlbaum methods and interventions. Mat

8 Hodson ME. Adults. In: Hodson ME, Geddes DM, eds. Cystic fibrosis London: Chapman and Hall Medical, 1995: 237-57.

9 Lanng S. Cystic fibrosis related diabetes mellitus. In: Book of abstracts, Fourth Australian and New Zealand Cystic Fibrosis Conference, Brisbane, Australia, 2001

10 Conway SP, Morton AM, Oldroyd B, et al. Osteoporosis and osteopenia in adults and adolescents with cystic fibrosis: prevalence and associated factors. Thorax 2000:55:798-804

11 Meichenbaum D, Turk DC. Treatment adherence: terminology, incidence and conceptualization. In: Facilitating treatment adherence. New York: Plenum Press, 1987: 19-39.

12 Cluss PA, Epstein LH. The measurement of medical compliance in the treatment of disease. In: Karoly $\mathrm{P}$, ed. Measurement strategies in health psychology. New York: John Wiley and Sons, 1985: 403-32.

13 Sackett DL, Snow JC. The magnitude of compliance and non-compliance. In: Haynes RB, Taylor DW, Sackett DL, eds. Compliance in health care. Baltimore: The Johns Hopkins University Press, 1979: $11-22$

14 Abbott J, Gee L. Contemporary psychosocial issues in cystic fibrosis: treatment adherence and quality of life. Disabil Rehabil 1998;20:662-71

15 Gordis L. Methodological issues in the measurement of patient compliance. In: Sackett DL, Haynes RB, eds. Compliance with therapeutic regimens. Baltimore: Johns Hopkins University Press, 1976. 
16 Lask B. Non-adherence to treatment in cystic fibrosis. J $R$ Soc Med 1994;87(Suppl 21):25-7.

17 Koocher GP, McGrath ML, Gudas L. Typologies of non-adherence in cystic fibrosis. J Dev Behav Pediatr 1990;11:353-8.

18 Epstein LH, Cluss PA. A behavioural medicine perspective on adherence to long-term medical regimens. J Consult Clin Psychol 1982;50:950-71.

19 Gordis L. Conceptual and methodologic problems in measuring patient compliance. In: Haynes RB, Taylor DW, Sackett DL, eds. Compliance in health care. Baltimore: The Johns Hopkins University Press, 1979: 23-45

20 Meyers A, Dolan TF, Mueller D. Compliance and self-medication in cystic fibrosis. Am J Dis Child 1975;129:1011-3.

21 Gong $\mathbf{H}$, Simmons MS, Clark VA, et al. Metered-dose inhaler usage in subjects with asthma: comparison of nebulizer chronolog and daily diary recordings. J Allergy Clin Immunol 1988;82:5-10.

22 Spector SL, Kinsman R, Mawhinney H, et al. Compliance of patients with asthma with an experimental aerosolized medication: implications for controlled clinical trials. J Allergy Clin Immunol 1986;77:65-70.

23 Rand CS, Wise RA, Nides $M$, et al. Metered-dose inhaler adherence in a clinical trial. Am Rev Respir Dis 1992;146:1559-64.

24 Starr M, Sawyer SM, Carlin JB, et al. A novel approach to monitoring adherence to preventive therapy for tuberculosis in adolescence. $J$ Paediatr Child Health 1999;35:350-4.

25 Chapman KR, Walker L, Cluley S, et al. Improving patient compliance with asthma therapy. Respir Med 2000;94:2-9.

26 Clark DM. Anxiety states: panic and generalized anxiety. In: Hawton K, Salkovskis PM, Kirk J, et al., eds. Cognitive behaviour therapy for psychiatric problems. A practical guide. New York: Oxford University Press, 1989: 52-96.

27 Bohannon NJ, Jack DB. Type II diabetes: tips for managing your older patients. Geriatrics 1996;51:28-35.

28 Chmielewski SA. Advances and strategies for glucose monitoring. Am J Clin Pathol 1995;4(Suppl 1):559-71.

29 Sawyer SM, Dapiran E. Patterns of adherence with pulmozyme in CF. Am J Respir Crit Care Med 2001;163:A639.

30 Lask B. Understanding and managing poor adherence in cystic fibrosis. Pediatr Pulmonol 1997; Suppl 16:260-1.

31 Sawyer SM, Blair S, Bowes G. Chronic illness in adolescents: transfer or transition to adult services? J Paediatr Child Health 1997:33:88-90.

32 Landau LI. Cystic fibrosis: transition from paediatric to adult physician's care. Thorax 1995;50:1031-2.

33 Patterson JM, Budd J, Goetz D, et al. Family correlates of a 10 year pulmonary health trend in cystic fibrosis. Pediatrics 1993;91:383-9.

34 Patterson JM, McCubbin HI, Warwick WJ. The impact of family functioning on health changes in children with cystic fibrosis. Soc Sci Med 1990;31:291-301.

35 Quittner AL, Tolbert VE, Regoli M, et al. Development of the role-play inventory of situations and coping strategies for parents of children with cystic fibrosis. J Pediatr Psychol 1996;21:209-35.
36 Conway SP, Pond MN, Hamnett T, et al. Compliance with treatment in adult patients with cystic fibrosis. Thorax 1996;51:29-33.

37 Abbott J, Dodd M, Bilton D, et al. Treatment compliance in adults with cystic fibrosis. Thorax 1994;49:115-20.

38 Conway SP, Pond MN, Watson A, et al. Knowledge of adult patients with cystic fibrosis about their illness. Thorax 1996;51:34-8.

39 Abbott J, Dodd M, Webb AK. Health perceptions and treatment adherence in adults with cystic fibrosis. Thorax 1996;51:1233-8

40 Rosenstock IM. Historical origins of the health belief model. Health Educ Monogr 1974: 2:328-35.

41 Wallston BS, Wallston KA. Locus of control and health: a review of the literature. Health Educ Monogr 1978;6:107-17.

42 Czajkowski DR, Koocher GP. Medical compliance and coping with cystic fibrosis. J Child Psychol Psychiatry 1987;28:311-9.

43 Bandura A. Social foundation of thought and action: a social cognitive theory. Englewood Cliffs, NJ: Prentice-Hall, 1986.

44 Parcel GS, Swank PR, Mariotto M, et al. Self-management of cystic fibrosis: a structural model for educational and behavioural variables. Soc Sci Med 1994;38:1307-15.

45 Leventhal H, Nerenz DR, Steele DJ. Illness representations and coping with health threats. In: Baum A, Taylor SE, Singer JE, eds. Handbook of psychology and health. Volume IV: Social psychological aspects of health. Hillsdale, NJ: Erlbaum, 1984: 219-52.

46 Weinman J, Petrie K, Moss-Morris R, et al. The illness perception questionnaire: a new method for assessing the cognitive representation of illness. Psychol Health 1996;11:431-45.

47 Petrie KJ, Weinman JA. Perceptions of health and illness. In: Petrie KJ, Weinman JA, eds. Perceptions of health and illness. Current research and applications. Amsterdam: Harwood Academic Publishers, 1997: $1-19$.

48 Horne R. Assessing perceptions of medications: psychological perspectives. In: McGavock H, ed. Handbook of drug research methodology. Newcastle upon Tyne: United Kingdom Drug Utilisation Research Group, 2000: 299-319.

49 Henley LD, Hill ID. Errors, gaps, and misconceptions in the disease-related knowledge of cystic fibrosis patients and their families. Pediatrics 1990;85: 1008-13.

50 levers CE, Brown RT, Drotar D, et al. Knowledge of physician prescriptions and adherence to treatment among children with cystic fibrosis and their mothers. J Dev Behav Pediatr 1999:20:335-43.

51 Petersen A. In a critical condition: health and power relations in Australia. St Leonards, NSW: Allen and Unwin, 1994: 154-6.

52 Thompson SM, Dahlquist LM, Koenning GM, et al. Brief report: adherence-facilitating behaviours of a multidisciplinary pediatric rheumatology staff. J Pediatr Psychol 1995;20:291-7.

53 Kettler L, Winefield H, Sawyer S, et al. Treatment perceptions and adherence in adults with cystic fibrosis. In: Book of abstracts, Fourth Australian and New Zealand Cystic Fibrosis Conference, Brisbane, Australia, 2001 